\title{
Estudio de 45 casos de cáncer del cuello uterino tratados quirúrgicamente en el Instituto Nacional de Cancerología
}

\begin{abstract}
Trabajo presentado a la Sociedad Colombiana de Obstetricia y Ginecología para ingreso como miembro de número por el doctor Germán Jordán Abondano.
\end{abstract}

\section{INTRODUCCION}

Se presenta el estudio detallado de 45 casos de carcinoma del cuello uterino, cuyas historias tomadas del archivo del Instituto Nacional de Cancerología pudieron ser estudiadas entre 50 que figuran como de enfermas tratadas con cirugía radical para este tipo de lesión, en el curso de cinco años comprendidos entre mayo de 1949 a abril de 1954. Las 5 historias no incluídas en este trabajo no fue posible encontrarlas en los archivos. La primera histerectomía radical con linfadenectomía tipo Wertheim Taussig practicada en el Instituto, se realizó en julio de 1949 actuando como cirujano el doctor Guillermo López Escobar.

No se hace un recuento detallado de la técnica quirúrgica, ni se pretende consignar detalles sobre clasificación, tratamiento, métodos, diagnósticos, metástasis, etc., porque todo esto ha sido presentado en miles de artículos de la literatura mundial.

A pesar del peligro de hacer pesado el trabajo para el lector, se presenta en un solo cuadro un resumen de todas las historias que permite a la vez una vista de conjunto y un estudio detallado de los casos estudiados. Posteriormente se concretan algunos de los datos de mayor interés.

\section{CONCEPTOS SOBRE CLASIFICACION DEL CANCER DE CUELLO UTERINO Y SU TRATAMIENTO QUIRURGICO}

La clasificación seguida en el Instituto Nacional de Cancerología es la adoptada internacionalmente por la Liga de las $\mathrm{Na}$ ciones en 1937 agregando el Carcinoma in-situ, clasificado como 
Estado O, de acuerdo con el Congreso Internacional de Obstetricia y Ginecología reunido en Nueva York en mayo de 1951.

No se incluyen en este trabajo los tratamientos quirúrgicos practicados en casos clasificados como Estado O, por no ser radical el tipo de intervención que se indica para ellos. Además en un estudio anterior el autor trató este tema.

Solamente se consideran como quirúrgicos los casos clasificados como en Estado I y II; y últimamente los miembros del servicio de cancelorogía en la especialidad ginecológica, de común acuerdo han resuelto en el Instituto no intervenir sino los clasificados en Estado I. No conviene extenderse sobre tratamiento quirúrgico post-irradiación ni de tipo más radical para casos avanzados o reproducidos, puesto que no se presenta ningún caso.

El tipo de intervención practicado en la mayoría de los casos es la histerectomía radical con linfadenectomía bilateral de los ganglios y tejido linfático y graso de las cadenas ilíaca externa, ilíaca interna y obturadora; esta linfadenectomía se practica en bloque de afuera hacia la línea media. En cuanto a la histerectomía, se inicia generalmente con disección del fondo de saco anterior, separando el peritoneo vésico-uterino, aislando totalmente el trayecto ureteral hasta su entrada en vejiga, ligando la arteria ilíaca interna (hipogástrica), disecando los dos tercios superiores de la vagina que se extraen y disecando para ligar contra la pelvis los parametrios laterales y posteriores (transversos y útero-sacros), sacando la pieza en bloque junto con los cos tercios superiores de vagina. La cúpula vaginal se sutura en Sli totalidad, posteriormente se peritoniza aislando pelvis de cavidad abdominal. Antes de disecar parametrios, se separa cuidadosamente peritoneo recto-uterino de útero y vagina. En los primeros casos se utilizó cateterismo ureteral previo, pero posteriormente se ha prescindido de éste.

Facilita la intervención el hacer taponamiento vaginal antes de iniciarla.

Varios autores han insistido sobre los factores que limitan el tratamiento quirúrgico del carcinoma del cuello uterino y no está por demás enumerar los principales: 1. La preparación y experiencia del cirujano; 2. Ei medio quirúrgico (anestesła, transfusión, instrumental, equipo de ayudantes, etc.); 3. Las conciciones generaies de la paciente; 4. La extensión del proceso tumoral. Cualquier intervención menos extensa que el tipo Wertheim Taussig no sólo es incompleta sino que generalmente ace- 
lera el proceso maligno y disminuye la posibilidad de un tratamiento apropiado por irradiaciones.

El estado general de la enferma: cardiovascular, renal, enfermedades del sistema nervioso, diabetes, obesidad pronunciada, tuberculosis y discrasia sanguínea, deben descartarse, pues son contraindicaciones del tratamiento quirúrgico.

El edema bilateral de los miembros, el que los ganglios paraórticos están invadidos o el hallazgo quirúrgico de fijación de vejiga a útero o de recto a útero o fijación de los parametrios a la pelvis, contraindican proseguir con la intervención y debe suturarse la herida abdominal remitiendo la enferma a tratamiento radioterápico.

\section{EXAMENES PREVIOS}

En toda enferma sometida a esta clase de cirugia deben practicarse de rutina los siguientes exámenes: cistoscopia, rectoscopia, examen clínico general y exámenes de laboratorio (orina, cuadro hemático. N. P. N., glicemia, tiempo de coagulación, sangría y protrombina). En el Instituto Nacional de Cancerología se realizan religiosamente estos exámenes antes de autorizar la intervención. 


\section{RESUMENES DE CASOS}

\begin{tabular}{|c|c|c|c|c|c|c|c|c|}
\hline $\begin{array}{l}\text { CASO } \\
\text { HIST. No } \\
\text { FECHA }\end{array}$ & $\begin{array}{l}\text { EDAD } \\
\text { PARID. } \\
\text { LAPSO } \\
\text { A } 10 \\
\text { CONSUL. }\end{array}$ & $\begin{array}{l}\text { ESTADO SINTO- } \\
\text { MATOLOGIA }\end{array}$ & BIOPSIAS & $\begin{array}{l}\text { PIEZA Q. } \\
\text { GANG. }\end{array}$ & $\begin{array}{l}\text { COMPLIC. } \\
\text { OPERAT. }\end{array}$ & TRATAMIENTOS & CONTROL & OBSERVACIONES \\
\hline $\begin{array}{l}\text { No } 1 \\
20771 \\
\text { V/49 }\end{array}$ & $\begin{array}{c}45 \\
5 \\
36 \text { mes. }\end{array}$ & $\begin{array}{l}\text { E: I } \\
\text { Hemorragias post } \\
\text { coitum. } \\
\text { Erosión cuello }\end{array}$ & $\begin{array}{c}\mathrm{V} / 49 \\
\mathrm{Ca} . \text { Esca- } \\
\text { moso }\end{array}$ & $\begin{array}{l}\text { Sin tumor } \\
\text { G: Negat. }\end{array}$ & NO & $\begin{array}{c}\text { VI/49: Radium. } \\
\text { VIII/49: Wer- } \\
\text { theim-T. }\end{array}$ & $\begin{array}{l}24 \text { meses } \\
\text { Sin Ca. } \\
\text { Aparente }\end{array}$ & $\begin{array}{l}\text { Epitelioma de endocervix tratado con Ra- } \\
\text { dium y Wertheim-T. Esterilización del Ca. } \\
\text { con la irradiación. Bien a las } 2 \text { años. Pér- } \\
\text { dida posteriormente de controi. }\end{array}$ \\
\hline $\begin{array}{l}\text { No } 2 \\
20869 \\
\text { VI/49 }\end{array}$ & $\begin{array}{c}38 \\
9 \\
10 \text { mes. }\end{array}$ & $\begin{array}{c}\text { E: I } \\
\text { Hemorragia pos } \\
\text { coitum. Flujo. } \\
\text { T. Exofítico. }\end{array}$ & $\begin{array}{l}\mathrm{VI} / 49 \\
\mathrm{Ca} . \text { Esca- } \\
\text { moso }\end{array}$ & $\begin{array}{l}\text { Ca. Escam. } \\
\text { G. Negat. }\end{array}$ & NO & $\begin{array}{l}\mathrm{VI} / 49: \text { Wer- } \\
\text { theim-T. }\end{array}$ & $\begin{array}{c}6 \text { meses } \\
\text { Reproduc. }\end{array}$ & $\begin{array}{l}\text { Cuatro meses después de la intervención } \\
\text { producción en tabique recto-vaginal y pa- } \\
\text { rametrios. Pérdida de control a los seis } \\
\text { meses. }\end{array}$ \\
\hline $\begin{array}{l}\text { No } 3 \\
21219 \\
\text { VII/49 }\end{array}$ & $\begin{array}{l}51 \\
9 \\
1 \text { mes. }\end{array}$ & $\begin{array}{c}\text { E: I } \\
\text { Erosión cuello } \\
\text { Embarazo? }\end{array}$ & $\begin{array}{c}\mathrm{VI} / 49 \\
\mathrm{Ca} \text {. Esca- } \\
\text { moso? } \\
\text { VII } / 49 \\
\text { Cervicitis } \\
\text { Sitolog. } \\
\text { Ca. ? }\end{array}$ & $\begin{array}{l}\text { Cervicitis } \\
\text { Endome- } \\
\text { triosis } \\
\text { G. Negat. }\end{array}$ & NO & $\begin{array}{l}\text { IX/49: Wer- } \\
\text { theim-T. }\end{array}$ & NO & $\begin{array}{l}\text { Sin control. Una biopsia fuera del Institu- } \\
\text { to diagnosticada equivocadamente como } \\
\text { Ca. Citologia, positiva para Ca. Pieza qui- } \\
\text { rúrgica negativa. Diagnóstico equivocado? }\end{array}$ \\
\hline $\begin{array}{l}\text { No } 4 \\
22340 \\
\text { I } / 50\end{array}$ & $\begin{aligned} & 32 \\
& 10 \\
4 & \text { mes. }\end{aligned}$ & $\begin{array}{c}\text { E: I } \\
\text { Metrorragia Pro- } \\
\text { lapso } 30 \mathrm{~g} \text {. } \\
\text { T. Exofítico. }\end{array}$ & $\begin{array}{c}\mathrm{I} / 50 \\
\mathrm{Ca} \quad \text { Esca- } \\
\text { moso }\end{array}$ & $\begin{array}{l}\overline{\text { Ca. Escam, }} \\
\text { G: No se } \\
\text { sacaron }\end{array}$ & $\begin{array}{c}\text { Shock } \\
\text { quirúrg. } \\
\text { reversible }\end{array}$ & $\begin{array}{l}\text { II/49: Hist. vagi- } \\
\text { nal Schauta } \\
\text { IX/52: Rx. pro- } \\
\text { fundos. }\end{array}$ & $\begin{array}{l}3 \text { meses } \\
\text { Reproduc. }\end{array}$ & $\begin{array}{l}\text { A los tres anos y siete meses reproduc- } \\
\text { ción en cúpula y ganglios ilíacos. La re- } \\
\text { producción tratada con rayos } x \text {. Sin con- } \\
\text { trol pusterior. }\end{array}$ \\
\hline $\begin{array}{l}\text { No } 5 \\
24593 \\
\mathrm{XI} / 50\end{array}$ & $\begin{array}{ll} & 45 \\
& 10 \\
3 & \text { mes. }\end{array}$ & $\begin{array}{l}\text { E: I } \\
\text { Flujo con sangre. } \\
\text { T. Exofítico. }\end{array}$ & $\begin{array}{c}\mathrm{XI} / 50 \\
\mathrm{Ca} \text { Esca- } \\
\text { moso }\end{array}$ & $\begin{array}{l}\text { Ca. Escam. } \\
\text { G: Negat. }\end{array}$ & $\begin{array}{l}\text { Snock post } \\
\text { operator. }\end{array}$ & $\begin{array}{c}\text { XII/50: Wer- } \\
\text { theim-T. }\end{array}$ & Muerte & $\begin{array}{l}\text { Murió diez horas después de la interven- } \\
\text { ción por shock quirúrgico. Antecedentes } \\
\text { de cardiopatia. }\end{array}$ \\
\hline $\begin{array}{l}\text { No } 6 \\
24869 \\
\text { XI/50 }\end{array}$ & 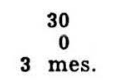 & $\begin{array}{l}\text { E: I } \\
\text { Polimenorreas } \\
\text { Erosión cuello }\end{array}$ & $\begin{array}{l}\mathrm{XI} / 50 \\
\text { Ca. Esca- } \\
\text { moso }\end{array}$ & $\begin{array}{l}\text { Ca. Escam. } \\
\text { G: Sin es- } \\
\text { tudio }\end{array}$ & No & $\begin{array}{c}\mathrm{XI} / 50: \text { Wer- } \\
\text { theim-T. }\end{array}$ & NO & No volvió a control. \\
\hline $\begin{array}{l}\text { No } 7 \\
25040 \\
\times I I / 50\end{array}$ & $\begin{array}{c}35 \\
9 \\
3 \text { mes. }\end{array}$ & $\begin{array}{l}\quad \text { E: I } \\
\text { Metrorragias } \\
\text { T. Exofitico. }\end{array}$ & $\begin{array}{l}\mathrm{XII} / 50 \\
\text { Ca. Esca- } \\
\text { moso }\end{array}$ & $\begin{array}{l}\text { Sin estu- } \\
\text { dio cuello. } \\
\text { G: Negat. }\end{array}$ & $\begin{array}{l}\text { Fístula } \\
\text { Vésico- } \\
\text { Vaginal }\end{array}$ & $\begin{array}{l}\text { I/51: Wer- } \\
\text { theim-T. }\end{array}$ & $\begin{array}{l}5 \text { meses } \\
\text { Sin Ca. } \\
\text { aparente }\end{array}$ & $\begin{array}{l}\text { Fístula vesico-vaginal tratada con catete- } \\
\text { rismo ureteral y placenta. Pérdida de con- } \\
\text { trol a los cinco meses. Sin cortes de cuello } \\
\text { en pieza quirúrgica. }\end{array}$ \\
\hline
\end{tabular}




\begin{tabular}{|c|c|c|c|c|c|c|c|c|}
\hline $\begin{array}{l}\text { CASO } \\
\text { HIST. N? } \\
\text { FECHA }\end{array}$ & $\begin{array}{l}\text { EDAD } \\
\text { PARID. } \\
\text { LAPSO } \\
\text { A } 10 \\
\text { CONSUL. }\end{array}$ & $\begin{array}{l}\text { ESTADO SINTO- } \\
\text { MATOLOGIA }\end{array}$ & BIOPSIAS & $\begin{array}{l}\text { PIEZA Q. } \\
\text { GANG. }\end{array}$ & $\begin{array}{l}\text { COMPLIC. } \\
\text { OPERAT. }\end{array}$ & TRATAMIENTOS & CONTROL & OBSERVACIONES \\
\hline $\begin{array}{c}\text { No } 8 \\
25083 \\
\text { I } / 51\end{array}$ & 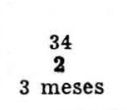 & $\begin{array}{l}\text { E: I } \\
\text { Menometrorragias } \\
\text { Erosión cuello. }\end{array}$ & $\begin{array}{l}\text { I/51 } \\
\text { Ca. Esca- } \\
\text { camoso. }\end{array}$ & $\begin{array}{l}\text { Mioma } \\
\text { Ca. Esc. } \\
\text { G: Negat. }\end{array}$ & No & $\begin{array}{l}\text { II/51: Wer- } \\
\text { theim } \mathrm{T} \text {. }\end{array}$ & $\begin{array}{l}35 \text { meses } \\
\text { Bien. Sin } \\
\text { Ca. apar. }\end{array}$ & Control de 3 tres años sin Ca. aparente. \\
\hline $\begin{array}{l}\text { No } 9 \\
25840 \\
\text { IV } / 51\end{array}$ & $\begin{array}{l}46 \\
11 \\
12 \\
\text { mes. }\end{array}$ & $\begin{array}{c}\text { E: I } \\
\text { T. Exofítico. } \\
\text { Hemorragia pos } \\
\text { coitum } \\
\text { Flujo con sangre. }\end{array}$ & $\begin{array}{l}\mathrm{IV} / 51 \\
\mathrm{Ca} / \text { Esca- } \\
\text { moso. }\end{array}$ & $\begin{array}{l}\text { Ca. Esca- } \\
\text { moso. } \\
\text { G: Sin } \\
\text { estudio }\end{array}$ & $\begin{array}{l}\text { Hemorra- } \\
\text { gia vena- } \\
\text { liaca. } \\
\text { Shock } \\
\text { Muerte. }\end{array}$ & $\begin{array}{c}\text { V/52: Wer- } \\
\text { theim } \mathrm{T} \text {. }\end{array}$ & Muerte. & $\begin{array}{l}\text { Menopausia de seis meses. Hemorragia } \\
\text { por lesión vena ilíaca externa tratada } \\
\text { con suturas. Shock operatorio. Muerte a } \\
\text { las } 4 \text { horas. Sin estudio ganglios. }\end{array}$ \\
\hline $\begin{array}{l}\text { No } 10 \\
25854 \\
\text { IV } / 51\end{array}$ & $\begin{array}{ll}55 \\
\\
3 & 11 \\
\text { mes. }\end{array}$ & $\begin{array}{l}\text { E: I } \\
\text { Hemorragia } \\
\text { Prolapso de } \\
\text { 30 grado } \\
\text { T. Exofítico }\end{array}$ & $\begin{array}{l}\text { IV/51 } \\
\text { Ca. Esca- } \\
\text { moso. } \\
\text { Cuello } \\
\text { amputado. }\end{array}$ & $\begin{array}{l}\text { Ca. Esc. } \\
\text { Propag. } \\
\text { Endomet. } \\
\text { Ca. Ovar. } \\
\text { Metastás. } \\
\text { G: Posti. }\end{array}$ & No & $\begin{array}{l}\text { VI/51: Wer- } \\
\text { theim T. } \\
\text { VII/51: Rx. } \\
\text { profundos. }\end{array}$ & $\begin{array}{l}39 \text { meses } \\
\text { Bien. Sin. } \\
\text { Ca. apar. }\end{array}$ & $\begin{array}{l}\text { Menopausia de cinco años. Control de tres } \\
\text { años y tres meses sin Ca, aparente. Am- } \\
\text { putación cuello y perineorrafia; al estu- } \\
\text { dio de cuello se encontró fuera del Insti- } \\
\text { tuto un Ca. escamoso. }\end{array}$ \\
\hline $\begin{array}{l}\text { No } 11 \\
25983 \\
\mathrm{~V} / 51\end{array}$ & $\begin{array}{c}36 \\
\\
3 \quad \text { mes. }\end{array}$ & $\begin{array}{c}\text { E: I } \\
\text { Hemorragias } \\
\text { pos coitum } \\
\text { Erosión cuello }\end{array}$ & $\frac{\begin{array}{c}\mathrm{V} / 51 \\
\mathrm{Ca} . \text { Esca- } \\
\text { moso. }\end{array}}{\mathrm{II} / 52}$ & $\begin{array}{l}\text { Mioma. } \\
\text { Ca. Esca- } \\
\text { moso. } \\
G: \text { Negat. }\end{array}$ & NO & $\begin{array}{c}\text { VI/51: Wer- } \\
\text { theim T. }\end{array}$ & $\begin{array}{l}12 \text { meses } \\
\text { Bien. Sin. } \\
\text { Ca. Apar. }\end{array}$ & $\begin{array}{l}\text { Dos años de metrorragias antes de pri- } \\
\text { mera consulta atribuíbles al mioma y no } \\
\text { al Ca. Hemorragia pos-coitum tres meses } \\
\text { antes de primera consulta. }\end{array}$ \\
\hline $\begin{array}{l}\text { No } 12 \\
27705 \\
\text { II } / 52\end{array}$ & $\begin{array}{c}33 \\
7 \\
2 \text { mes. } \\
\end{array}$ & $\begin{array}{l}\text { E: I } \\
\text { Flujo fétido } \\
\text { con sangre } \\
\text { T. Exofítico }\end{array}$ & $\begin{array}{c}\begin{array}{c}\text { Ca. Atí- } \\
\text { pico. }\end{array} \\
\text { III/52 } \\
\text { Ca. de }\end{array}$ & $\begin{array}{l}\text { Adeno Ca. } \\
\text { lel cuello. } \\
\text { G: Sin es- } \\
\text { tudio }\end{array}$ & No & $\begin{array}{c}\text { II } / 52: \text { Wer- } \\
\text { theim } \mathrm{T} .\end{array}$ & NO & $\begin{array}{l}\text { No volvió a control. Adeno-Ca. de cuello } \\
\text { con endometrio normal. Sin estudio gan- } \\
\text { glios. }\end{array}$ \\
\hline $\begin{array}{l}\text { No } 13 \\
27844 \\
\text { III } / 52\end{array}$ & $\begin{array}{l}45 \\
8 \\
36 \\
\text { mes. }\end{array}$ & $\begin{array}{l}\text { Hemorragia pos } \\
\text { coitum. } \\
\text { Pólipos degene- } \\
\text { rados. }\end{array}$ & $\frac{\begin{array}{c}\text { transi- } \\
\text { ción. }\end{array}}{\text { IV/52 }}$ & $\begin{array}{l}\text { Ca. de } \\
\text { Transic. } \\
\text { Muñón } \\
\text { cuello } \\
\text { ix: Negat. }\end{array}$ & No & $\begin{array}{l}\text { III/52: Wertheim } \\
\text { T. sobre muñón y } \\
\text { anexos residuales. }\end{array}$ & $\begin{array}{l}15 \text { meses } \\
\text { Bien. Sin. } \\
\text { Ca. Apar. }\end{array}$ & $\begin{array}{l}\text { Se abrió y saturó vejiga durante la in } \\
\text { tervención. Histerectomía sub-total tres } \\
\text { años antes. Hemorragia pos-coitum des- } \\
\text { pués de la intervención. }\end{array}$ \\
\hline $\begin{array}{l}\text { No } 14 \\
27946 \\
\text { IV } / 52\end{array}$ & $\begin{array}{ll}30 \\
10 \\
2 \text { mes. }\end{array}$ & $\begin{array}{l}\text { E: I } \\
\text { Meno-metrorragias } \\
\text { T. Exofítico. }\end{array}$ & $\begin{array}{l}\text { moso. } \\
\text { IV } / 52 \\
\text { Ca. Esca- } \\
\text { moso. }\end{array}$ & $\begin{array}{l}\text { 2a. Esca- } \\
\text { moso. } \\
\text { G: Negat. }\end{array}$ & No & $\begin{array}{l}\text { IV/52: Wer- } \\
\text { sub-peritoneal. }\end{array}$ & $\begin{array}{l}18 \text { meses } \\
\text { Bien. Sin. } \\
\text { Ca. apar. }\end{array}$ & . \\
\hline
\end{tabular}




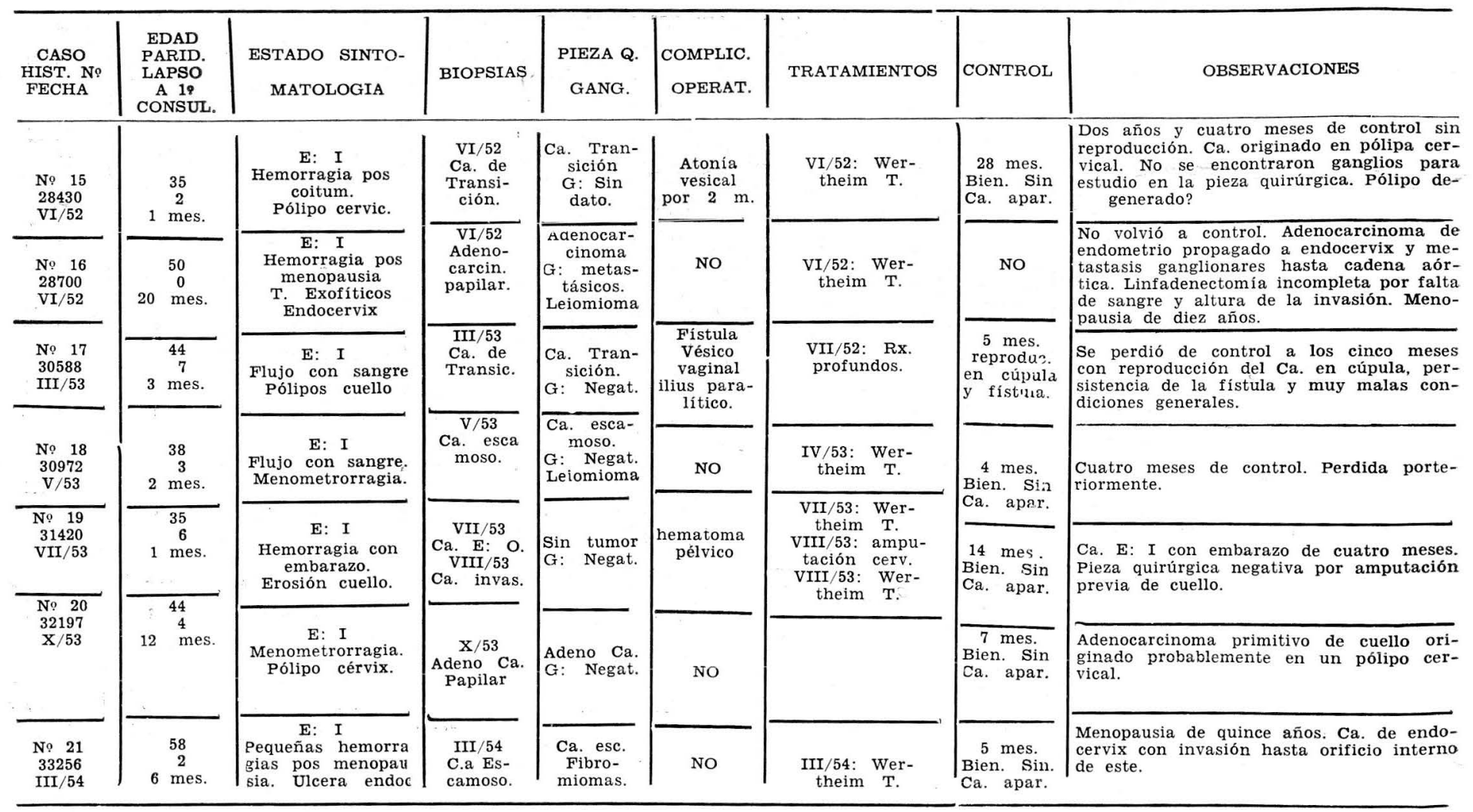




\begin{tabular}{|c|c|c|c|c|c|c|c|c|}
\hline $\begin{array}{l}\text { CASO } \\
\text { HIST. No } \\
\text { FECHA }\end{array}$ & $\begin{array}{l}\text { EDAD } \\
\text { PARID. } \\
\text { LAPSO } \\
\text { A 10 } \\
\text { CONSUL. }\end{array}$ & $\begin{array}{l}\text { ESTADO SINTO- } \\
\text { MATOLOGIA }\end{array}$ & BIOPSIAS & $\begin{array}{l}\text { PIEZA Q. } \\
\text { GANG. }\end{array}$ & $\begin{array}{l}\text { COMPLIC. } \\
\text { OPERAT. }\end{array}$ & TRATAMIENTOS & CONTROL & OBSERVACIONES \\
\hline $\begin{array}{l}\text { No } 22 \\
33715 \\
\text { IV } / 54\end{array}$ & $\begin{array}{c}34 \\
6 \\
6 \text { mes. }\end{array}$ & $\begin{array}{l}\text { E: I } \\
\text { Flujo maloliente. } \\
\text { Purulento. Gran i } \\
\text { Exofítico. }\end{array}$ & $\begin{array}{l}\text { IV } / 54 \\
\text { Ca. es- } \\
\text { camoso. }\end{array}$ & $\begin{array}{l}\text { Ca. esca- } \\
\text { moso. } \\
\text { Ganflios } \\
\text { negativos. }\end{array}$ & $\begin{array}{l}\text { Abceso } \\
\text { colibacilar } \\
\text { de la he- } \\
\text { rida }\end{array}$ & $\begin{array}{c}\text { V/54: } \\
\text { Wer- } \\
\text { theim } \mathrm{T} \text {. }\end{array}$ & $\begin{array}{l}4 \text { mes. } \\
\text { Bien. Sin } \\
\text { Ca. apar. }\end{array}$ & $\begin{array}{l}\text { Abceso de la pared por colibacilo. Curó } \\
\text { fácilmente. Gran tumor necrótico en co- } \\
\text { liflor. }\end{array}$ \\
\hline $\begin{array}{l}\text { No } 23 \\
22240 \\
\mathrm{XII} / 49\end{array}$ & $\begin{array}{c}50 \\
4 \\
3 \text { mes. }\end{array}$ & $\begin{array}{l}\text { E: II } \\
\text { Vagina. Paramet. } \\
\text { Hemorragias pos. } \\
\text { Menopausia. } \\
\text { Nódulo parametria: }\end{array}$ & $\begin{array}{l}\text { XII/49 } \\
\text { Adenocar- } \\
\text { cinoma del } \\
\text { cuello. }\end{array}$ & $\begin{array}{l}\text { Negativa } \\
\text { para Ca. } \\
\text { G: Negat. } \\
\text { Mioma. } \\
\text { Salpingitis }\end{array}$ & $\begin{array}{l}\text { Parálisis } \\
\text { de brazo } \\
\text { d. Inconti- } \\
\text { nencia } \\
\text { uretral. }\end{array}$ & $\begin{array}{c}\text { I/50: radium pre- } \\
\text { cervical y sonda. } \\
\text { III } / 50: \text { Wer- } \\
\text { theim } \mathrm{T} \text {. }\end{array}$ & $\begin{array}{l}53 \text { mes. } \\
\text { Bien. Sin } \\
\text { Ca. apar. }\end{array}$ & $\begin{array}{l}\text { Menopausia de un año. Adenocarcinoma } \\
\text { del cuello; no se sabe si también del } \\
\text { cuerpo, pues el tratamiento previo con } \\
\text { radium esterilizó el Ca. La pieza quirúr- } \\
\text { gica negativa para Ca. Control cuatro } \\
\text { años y cinco meses. }\end{array}$ \\
\hline $\begin{array}{c}\text { No } 24 \\
22263 \\
\text { XII } / 49\end{array}$ & $\begin{array}{ll} & 76 \\
& 10 \\
6 & \text { mes. }\end{array}$ & $\begin{array}{c}\text { E: II } \\
\text { Hemorragia pos } \\
\text { menopausia. T. } \\
\text { Exofítico. Vagina } \\
\text { parametrios. } \\
\text { Prolapso } 30 \mathrm{G} \text {. }\end{array}$ & $\begin{array}{l}\text { XII/ } 49 \\
\text { Ca. Esca- } \\
\text { moso. }\end{array}$ & $\begin{array}{l}\text { Ca. esca- } \\
\text { moso. } \\
\text { G: no se } \\
\text { sacaron. }\end{array}$ & $\begin{array}{l}\text { Shock } \\
\text { operatorio } \\
\text { reversible. }\end{array}$ & $\begin{array}{l}\text { I/50: histerer- } \\
\text { tomía vaginal } \\
\text { Schauta }\end{array}$ & 1 mes. & $\begin{array}{l}\text { Menopausia de ventidós años. Prolapso } \\
\text { total. No se practicó linfadenectomía. } \\
\text { Perdida de control. }\end{array}$ \\
\hline $\begin{array}{l}\text { No } 25 \\
22842 \\
\text { III } / 50\end{array}$ & $\begin{array}{l}28 \\
6 \text { mes. } \\
6 \text { mes. }\end{array}$ & $\begin{array}{c}\text { E: II } \\
\text { Vagina. Flujo ca } \\
\text { sangre. Hemorragi } \\
\text { pos coitum. Cuell } \\
t \text { umoral. Duro }\end{array}$ & $\begin{array}{l}\mathrm{III} / 50 \\
\mathrm{Ca} . \text { esca- } \\
\text { moso. }\end{array}$ & $\begin{array}{l}\text { Ca. esca- } \\
\text { moso. } \\
\text { G: negat. }\end{array}$ & No & $\begin{array}{cc}\text { III/50: } & \text { Wer- } \\
\text { theim } & \text { T. }\end{array}$ & $\begin{array}{l}55 \text { mes. } \\
\text { Bien. Sin } \\
\text { Ca. apar. }\end{array}$ & $\begin{array}{l}\text { Cuatro años y siete meses de control sin } \\
\text { signos de reproducción. }\end{array}$ \\
\hline $\begin{array}{l}\text { No } 26 \\
23030 \\
\text { IV } / 50\end{array}$ & $\begin{array}{ll} & 40 \\
4 & \text { mes. } \\
4 & \text { mes. }\end{array}$ & $\begin{array}{l}\text { E: II } \\
\text { Parametrios } \\
\text { T. Exofitico } \\
\text { cuello } \\
\text { Ca. endometrio }\end{array}$ & $\begin{array}{l}\text { IV } / 50 \\
\text { Adeno Ca. } \\
\text { del cuello. }\end{array}$ & $\begin{array}{l}\text { Adeno Ca. } \\
\text { de endo- } \\
\text { metrio. } \\
\text { Cuello: } \\
\text { sin T. } \\
\text { G: no se } \\
\text { sacaron. }\end{array}$ & NO & $\begin{array}{l}\text { IV/50: radium pre- } \\
\text { cervical y sonda } \\
\text { intraut. } \\
\text { VI/50: Wer- } \\
\text { theim (sin lin- } \\
\text { fadenect). }\end{array}$ & $\begin{array}{l}37 \text { mes. } \\
\text { Bien. Sin. } \\
\text { Ca. apar. }\end{array}$ & $\begin{array}{l}\text { Adenocarcinoma de endometrio propagado } \\
\text { a cuello tratado con sonda intrauterina } \\
\text { de radium y radium precervical. En pieza } \\
\text { quirúrgica Ca. residual de endometrio y } \\
\text { cuello sin tumor. No se practicó linfade- } \\
\text { nectomía. Control postoperatorio } 35 \text { meses } \\
\text { y } 37 \text { después del radium. }\end{array}$ \\
\hline
\end{tabular}




\begin{tabular}{|c|c|c|c|c|c|c|c|c|}
\hline $\begin{array}{l}\text { CASO } \\
\text { HIST. No } \\
\text { FECHA }\end{array}$ & $\begin{array}{l}\text { EDAD } \\
\text { PARID. } \\
\text { LAPSO } \\
\text { A 10. } \\
\text { CONSUL. }\end{array}$ & $\begin{array}{l}\text { ESTADO SINTO- } \\
\text { MATOLOGIA }\end{array}$ & BIOPSIAS & $\begin{array}{l}\text { PIEZA Q. } \\
\text { GANG. }\end{array}$ & $\begin{array}{l}\text { COMPLIC. } \\
\text { OPERAT. }\end{array}$ & TRATAMIENTOS & CONTROL & OBSERVACIONES \\
\hline $\begin{array}{l}\text { No } 27 \\
23143 \\
\text { IV } / 50\end{array}$ & $\begin{array}{c}30 \\
1 \\
6 \text { mes. }\end{array}$ & $\begin{array}{l}\text { E: II } \\
\text { Parametrios. } \\
\text { T. Exofítico. } \\
\text { Salpingitis. } \\
\text { Metrorragias }\end{array}$ & $\begin{array}{l}\text { IV } / 50 \\
\text { Ca. Esca- } \\
\text { moso. }\end{array}$ & $\begin{array}{l}\text { Ca. Esca- } \\
\text { moso. } \\
\text { Galpingitis } \\
\text { G: Sin es- }\end{array}$ & $\begin{array}{l}\text { Cicatriza- } \\
\text { ción in- } \\
\text { completa } \\
\text { de cúpula } \\
\text { vaginal }\end{array}$ & $\begin{array}{l}\text { V/50: Wer- } \\
\text { theim T. } \\
\text { X/50: Rayos } \mathbf{x} \\
\text { Tratamiento } \\
\text { incompleto. }\end{array}$ & $\begin{array}{l}5 \text { mes. } \\
\text { reproduc. } \\
8 \text { mes. } \\
\text { Muerte }\end{array}$ & $\begin{array}{l}\text { El cirujano sospechó que los ganglios eran } \\
\text { metastásicos. No se practicaron cortes de } \\
\text { ellos. Reproducción a los cinco meses en } \\
\text { cúpula y parametrios tratada con rayos } \\
\text { X. Abandonó tratamiento. Muerte a los } \\
\text { ocho meses. }\end{array}$ \\
\hline $\begin{array}{l}\text { No } 28 \\
23793 \\
\text { VII } / 50\end{array}$ & $\begin{array}{l}43 \\
13 \\
4 \text { mes. }\end{array}$ & $\begin{array}{l}\text { E: II } \\
\text { Vag. Parametrios } \\
\text { Flujo con sangre } \\
\text { T. Exofitico. }\end{array}$ & $\begin{array}{l}\text { VII } / 50 \\
\text { Ca. Esca- } \\
\text { moso. }\end{array}$ & $\begin{array}{l}\text { tudio. } \\
\mathrm{Ca} \text {. Esca- } \\
\text { moso. } \\
\mathrm{G}: \text { positi- } \\
\text { vos. }\end{array}$ & $\begin{array}{l}\text { Paresia } \\
\text { brazo } \\
\text { derecho }\end{array}$ & $\begin{array}{c}\text { VII /50: Wer- } \\
\text { theim T. }\end{array}$ & $\begin{array}{l}48 \text { mes. } \\
\text { 3ien. Sin } \\
\text { Ca. apar. }\end{array}$ & $\begin{array}{l}\text { Control de cuatro años sin Ca. aparente a } \\
\text { pesar de ganglios positivos. La paresia } \\
\text { del brazo derecho curó a los cinco meses. }\end{array}$ \\
\hline $\begin{array}{l}\text { No } 29 \\
27187 \\
\mathrm{X} / 51\end{array}$ & $\begin{array}{c}42 \\
2 \\
2 \\
\text { mes. }\end{array}$ & $\begin{array}{l}\text { E: II } \\
\text { Vag. Parametrios } \\
\text { Erosión cuelo } \\
\text { (endofítico) } \\
\text { Metrorragias. }\end{array}$ & $\begin{array}{l}\mathrm{X} / 51 \\
\mathrm{Ca} \cdot \mathrm{Esca-} \\
\text { moso. }\end{array}$ & $\begin{array}{l}\text { Pieza Q. } \\
\text { y ganglio } \\
\text { sin des- } \\
\text { cripción. }\end{array}$ & $\begin{array}{l}\text { Fístula } \\
\text { uretero } \\
\text { vaginal } \\
\text { Necrosis } \\
\text { cúpula. }\end{array}$ & $\begin{array}{l}\text { V/52: Reparación } \\
\text { fístula. }\end{array}$ & $\begin{array}{l}8 \text { mes. } \\
\text { Bien. Sin } \\
\text { Ca. ni } \\
\text { fístula }\end{array}$ & $\begin{array}{l}\text { Fístula uretero-vaginal curada quirúrgica- } \\
\text { mente por reimplantación del uretero en } \\
\text { vejiga a los siete meses del wertheim. } \\
\text { Pérdida después para control. }\end{array}$ \\
\hline $\begin{array}{l}\text { No } 30 \\
27467 \\
\mathrm{XII} / 51\end{array}$ & $\begin{array}{c}47 \\
\quad \\
8 \\
8 \text { mes. }\end{array}$ & $\begin{array}{l}\text { E: II } \\
\text { Parametrios. } \\
\text { Polimenorreas } \\
\text { T. Exofítico } \\
\text { del cuello. }\end{array}$ & $\begin{array}{l}\mathrm{XI} / 51 \\
\text { Adeno Ca. } \\
\text { Papili- } \\
\text { fero. }\end{array}$ & $\begin{array}{l}\text { Sin tumor. } \\
\text { G: no se } \\
\text { extirparon. }\end{array}$ & NO & $\begin{array}{l}\text { I/52: } \text { Rayos } X \\
\text { y radium. } \\
\text { I/54: Wer- } \\
\text { theim Simple. }\end{array}$ & $\begin{array}{l}32 \text { mes. } \\
\text { Pos-Rx } \\
8 \text { mes. } \\
\text { pos-Wer- } \\
\text { Theim. } \\
\text { Bien. }\end{array}$ & $\begin{array}{l}\text { Wertheim simple dos años después de irra- } \\
\text { diada. Sospecha clínica de Ca. en pieza } \\
\text { quirúrgica, pero cortes inapropiados. Con- } \\
\text { trol pos-operatorio ocho meses. }\end{array}$ \\
\hline $\begin{array}{l}\text { No } 31 \\
27857 \\
\text { II } / 52\end{array}$ & $\begin{array}{l}56-7 \\
1 \text { mes. } \\
34\end{array}$ & $\begin{array}{l}\text { E: II } \\
\text { Parametrios. } \\
\text { Cráter cuello } \\
\text { (T. endofitico) } \\
\text { Metrorragias. }\end{array}$ & $\begin{array}{l}\text { II } / 52 \\
\text { Ca. Esca- } \\
\text { moso. }\end{array}$ & $\begin{array}{l}\text { Ca. de } \\
\text { Transa- } \\
\text { ción. } \\
\text { G: Negat. }\end{array}$ & $\begin{array}{l}\text { Lesión } \\
\text { novena } \\
\text { iliaca } \\
\text { sutura }\end{array}$ & $\begin{array}{cl}\text { III/52: } & \text { Wer- } \\
\text { theim } & \text { T. }\end{array}$ & $\begin{array}{l}24 \text { mes. } \\
\text { Muerte. }\end{array}$ & $\begin{array}{l}\text { Menopausia de die zaños. Reproducción a } \\
\text { los siete meses en tabique recto-vaginal. } \\
\text { No aceptó tratamiento por rayos } X \text {. Muer- } \\
\text { te a los dos años por carcinoma. }\end{array}$ \\
\hline $\begin{array}{l}\text { No } 32 \\
28364 \\
\text { IV } / 52\end{array}$ & $\begin{array}{c}34 \\
\quad 2 \\
6 \text { mes. }\end{array}$ & $\begin{array}{l}\text { E: II } \\
\text { Vagina. Parame- } \\
\text { trios. T. Endofíti- } \\
\text { co de muñón de } \\
\text { cuello. Hemorra- } \\
\text { gia pos coitum. }\end{array}$ & $\begin{array}{c}\text { IV } / 52 \\
\text { Ca. Esca- } \\
\text { moso. }\end{array}$ & $\begin{array}{l}\text { Ca. esca- } \\
\text { moso. } \\
\text { G: Negat. }\end{array}$ & NO & $\begin{array}{l}\text { IV/52: Estracciói } \\
\text { de muñón. Linfı } \\
\text { denectomía. }\end{array}$ & $\begin{array}{l}\text { Muerte. } \\
\text { sin con- } \\
\text { troles in- } \\
\text { termedios } \\
\text { a los } 12 \\
\text { mes. }\end{array}$ & $\begin{array}{l}\text { Histerectomía subtotal die zaños antes. } \\
\text { Muerte al año. No se supo si por carci- } \\
\text { nomatosis. Ca. de muñón de cuello re- } \\
\text { sidual. }\end{array}$ \\
\hline
\end{tabular}




\begin{tabular}{|c|c|c|c|c|c|c|c|c|}
\hline $\begin{array}{l}\text { CASO } \\
\text { HIST. N? } \\
\text { FECHA }\end{array}$ & $\begin{array}{l}\text { EDAD } \\
\text { PARID. } \\
\text { LAPSO } \\
\text { A } 19 \\
\text { CONSUL. }\end{array}$ & $\begin{array}{c}\text { ESTADO SINTO- } \\
\text { MATOLOGIA }\end{array}$ & BIOPSIAS & $\begin{array}{l}\text { PIEZA Q. } \\
\text { GANG. }\end{array}$ & $\begin{array}{l}\text { OPERAT. } \\
\text { COMPLIC. }\end{array}$ & TRATAMIENTOS & CONTROL & OBSERVACIONES \\
\hline $\begin{array}{l}\text { No } 33 \\
28417 \\
\mathrm{~V} / 52\end{array}$ & $\begin{array}{c}32 \\
3 \\
2 \text { mes. }\end{array}$ & $\begin{array}{c}\text { E: II } \\
\text { Parametrios } \\
\text { Pequeñas hemo- } \\
\text { rragias. } \\
\text { T. Exofítico. } \\
\text { Salpingitis }\end{array}$ & $\begin{array}{l}\text { V/52 } \\
\text { Ca. Epi- } \\
\text { dermoide } \\
\text { escamoso. }\end{array}$ & $\begin{array}{l}\text { Cortes } \\
\text { inapropia- } \\
\text { dos. No se } \\
\text { encontró } \\
\text { Ca. } \\
\text { G: Negat. } \\
\text { Salpingitis. }\end{array}$ & $\begin{array}{l}\text { Sinock } \\
\text { quirúrgico } \\
\text { por hemo- } \\
\text { rragia } \\
\text { fístula } \\
\text { vésicova- } \\
\text { ginal. } \\
\text { eeritonitis. }\end{array}$ & $\begin{array}{l}\text { VI/52: Wer- } \\
\text { theim T. }\end{array}$ & $\begin{array}{l}2 \text { mes. } \\
\text { reproduc- } \\
\text { ción en } \\
\text { cúpula? } \\
\text { Mal. }\end{array}$ & $\begin{array}{l}\text { Salpingitis sub-aguda en el tiempo de la } \\
\text { intervención. Fue necesario dejar tapona- } \\
\text { miento pélvico tipo Mickulicz por hemo- } \\
\text { rragia. Fístula vésico-vaginal. Peritonitis } \\
\text { pos-operatoria. Pérdida de control a los } \\
\text { dos meses con sospecha de reproducción } \\
\text { en cúpula y en malas condiciones gene- } \\
\text { rales. }\end{array}$ \\
\hline $\begin{array}{c}\text { No } 34 \\
28904 \\
\text { VII } / 52\end{array}$ & $\begin{array}{c}37 \\
6 \\
6 \text { mes. }\end{array}$ & $\begin{array}{l}\text { E: II } \\
\text { Parametrios } \\
\text { Flujo con sangre. } \\
\text { T. Exofítico. }\end{array}$ & $\begin{array}{l}\text { VII } / 52 \\
\text { Ca. de } \\
\text { Transi- } \\
\text { ción. }\end{array}$ & $\begin{array}{l}\text { Ca. de } \\
\text { Transi- } \\
\text { sión. } \\
\text { G: Negat. }\end{array}$ & No & $\begin{array}{l}\text { VII/52 Wer- } \\
\text { theim T. } \\
\text { II } / 53: \text { Estración } \\
\text { metast. vaginal } \\
\text { V/53: rayos X } \\
\text { profundos. }\end{array}$ & $\begin{array}{l}8 \text { mes. } \\
\text { reproduc. } \\
12 \text { mes. } \\
\text { invasión } \\
\text { pélvica. }\end{array}$ & $\begin{array}{l}\text { Reproducción en cúpula y para metrios a } \\
\text { los ocho meses (tratada con Rx.). En } \\
\text { control con invasión pélvica. }\end{array}$ \\
\hline $\begin{array}{l}\text { No } \quad 35 \\
29349 \\
\mathrm{IX} / 52\end{array}$ & $\begin{array}{ll} & 48 \\
& 12 \\
3 & \text { mes. }\end{array}$ & $\begin{array}{c}\text { E: II } \\
\text { Parametrios. } \\
\text { Flujo con sangri } \\
\text { Placas de leucopla- } \\
\text { sia cuello. } \\
\text { T. Exofitico. }\end{array}$ & $\begin{array}{l}\mathrm{IX} / 52 \\
\mathrm{Ca} \text {. esca- } \\
\text { moso. }\end{array}$ & $\begin{array}{l}\text { Ca. esca- } \\
\text { moso. } \\
\text { G: Negat. }\end{array}$ & $\begin{array}{l}\text { Shock } \\
\text { huirúrgico } \\
\text { irreversi- } \\
\text { ble. } \\
\text { Muerte. }\end{array}$ & $\begin{array}{l}\text { IX/52: Wer- } \\
\text { theim T. }\end{array}$ & Muerte & $\begin{array}{l}\text { Murió en la sala quirúrgica por shock } \\
\text { operatorio. Enferma obesa. }\end{array}$ \\
\hline $\begin{array}{l}\text { No } 36 \\
29510 \\
\mathrm{X} / 52\end{array}$ & $\begin{array}{c}52 \\
2 \\
2 \\
\text { mes. }\end{array}$ & $\begin{array}{c}\text { E: II } \\
\text { Parametrios. } \\
\text { Cráter del cérvis } \\
\text { (endofítico). } \\
\text { Miomas utero. }\end{array}$ & $\begin{array}{l}\mathrm{X} / 52 \\
\text { Ca. Esca- } \\
\text { moso con } \\
\text { zonas a- } \\
\text { naplásicas. }\end{array}$ & $\begin{array}{l}\text { Ca. espi- } \\
\text { noso. } \\
\text { G: } \text { Negat. }\end{array}$ & $\begin{array}{l}\text { Shock a } \\
\text { las } 24 \text { h. } \\
\text { eventra- } \\
\text { ción. } \\
\text { Netritis } \\
\text { Muerte }\end{array}$ & $\begin{array}{l}\mathrm{X} / 52: \text { Wer- } \\
\text { theim } \mathrm{T} \text {. }\end{array}$ & $\begin{array}{l}\text { Muerte a } \\
\text { los } 10 \\
\text { días. }\end{array}$ & $\begin{array}{l}\text { Menopausia de seis meses. Shock quirúr- } \\
\text { fico tardio. Dehiscencia de planos pro- } \\
\text { undos de la herida. Muerte a los diez } \\
\text { Pías. Autopsia, edema cerebral. Nefritis. }\end{array}$ \\
\hline
\end{tabular}




\begin{tabular}{|c|c|c|c|c|c|c|c|c|}
\hline $\begin{array}{l}\text { CASO } \\
\text { HIST. No } \\
\text { FECHA }\end{array}$ & $\begin{array}{l}\text { EDAD } \\
\text { PARID. } \\
\text { LAPSO } \\
\text { A } 10 \\
\text { CONSUL. }\end{array}$ & $\begin{array}{c}\text { ESTADO SINTO- } \\
\text { MATOLOGIA }\end{array}$ & BIOPSIAS & $\begin{array}{l}\text { PIEZA Q. } \\
\text { GANG. }\end{array}$ & $\begin{array}{l}\text { COMPLIC. } \\
\text { OPERAT. }\end{array}$ & TRATAMIENTOS & CONTROL & OBSERVACIONES \\
\hline $\begin{array}{l}\text { No } 37 \\
29770 \\
\mathrm{XI} / 52\end{array}$ & $\begin{array}{c}52 \\
5 \\
6 \text { mes. }\end{array}$ & $\begin{array}{l}\text { E: II } \\
\text { Vagina-Param. } \\
\text { Hemorragia pos } \\
\text { menopausia. } \\
\text { T. Exofitico. }\end{array}$ & $\begin{array}{l}\mathrm{IX} / 53 \\
\mathrm{Ca} \text {. esca- } \\
\text { moso. } \\
\text { V/53 } \\
\text { Ca. Esca. } \\
\text { de Fistul. }\end{array}$ & $\begin{array}{l}\text { Ca. Epi- } \\
\text { cermoide. } \\
\text { G: Posit. } \\
\text { Paramet. } \\
\text { Posit. Ca. } \\
\text { V/53 Ca. } \\
\text { Epider- } \\
\text { moide. }\end{array}$ & $\begin{array}{l}\text { Fístula } \\
\text { vésicova- } \\
\text { ginal de- } \\
\text { hiscencia } \\
1 / 3 \text { planos } \\
\text { sup. heri. }\end{array}$ & $\begin{array}{l}\text { I/55: Wer- } \\
\text { theim T. } \\
\text { V253: Cierre } \\
\text { quirúrgico } \\
\text { fístula. }\end{array}$ & $\begin{array}{l}5 \text { mes. } \\
\text { muerte }\end{array}$ & $\begin{array}{l}\text { Uenopausia de dós años. Fistula vésico- } \\
\text { raginal operada a los cuatro meses con } \\
\text { a. en los bordes. Muerte a los cinco } \\
\text { leses con fístula reproducción y caque- } \\
\text { zia. Ruptura y sutura de vejiga en in- } \\
\text { tervención. }\end{array}$ \\
\hline $\begin{array}{l}\text { Noo } 38 \\
29783 \\
\text { XI } / 52\end{array}$ & $\begin{array}{cc} & 56 \\
& 7 \\
3 & \mathrm{mes} .\end{array}$ & $\begin{array}{c}\text { E: II } \\
\text { Vagina. } \\
\text { Cráter endofí. } \\
\text { Hemor. pos-meno. }\end{array}$ & $\begin{array}{c}\mathrm{XI} / 52 \\
\mathrm{Ca} . \text { Esca- } \\
\text { moso. }\end{array}$ & $\begin{array}{l}\text { Ca. Epi- } \\
\text { Alermoide. } \\
\text { G: Negat. }\end{array}$ & NO & $\begin{array}{c}\text { XII/52: Wer- } \\
\text { theim T. }\end{array}$ & $\begin{array}{l}12 \text { mes. } \\
\text { Bien Sin } \\
\text { Ca. apsr. }\end{array}$ & $\begin{array}{l}\text { Pequeña eventración a los seis meses en } \\
\text { herida abdominal. }\end{array}$ \\
\hline $\begin{array}{l}\text { No } 39 \\
30022 \\
\mathrm{XI} / 52\end{array}$ & $\begin{array}{ll} & 44 \\
& 17 \\
3 & \text { mes. }\end{array}$ & $\begin{array}{l}\text { E: II } \\
\text { Vagina. } \\
\text { Metrorragia. } \\
\text { Hemor. post-coit } \\
\text { Flujo hemático. } \\
\text { T. Exofítico. }\end{array}$ & $\begin{array}{l}\text { XII } / 53 \\
\text { Ca. Esca- } \\
\text { moso. }\end{array}$ & $\begin{array}{l}\text { Ca. Epid. } \\
\text { G: posit. } \\
\text { Paramet. } \\
\text { Positivos } \\
\text { para Ca. }\end{array}$ & $\begin{array}{l}\text { Parálisis } \\
\text { vesical. }\end{array}$ & $\begin{array}{l}\text { I/53: Wer- } \\
\text { theim } \mathrm{T} \text {. }\end{array}$ & NO & $\begin{array}{l}\text { Perdida de control. Parálisis vesical en } \\
\text { los veinte primeros días de pos-operatorio. }\end{array}$ \\
\hline $\begin{array}{l}\text { No } 40 \\
30080 \\
\mathrm{X} / 53\end{array}$ & $\begin{array}{c}55 \\
9 \\
6 \text { mes. }\end{array}$ & $\begin{array}{l}\text { E: II } \\
\text { Vag. Paramet. } \\
\text { Hemorragias. } \\
\text { posmenopausia }\end{array}$ & $\begin{array}{l}\mathrm{I} / 53 \\
\text { Ca. de } \\
\text { Transi- }\end{array}$ & $\begin{array}{l}\text { a. esca- } \\
\text { moso. } \\
\text { v: positi- } \\
\text { vos para } \\
\text { Ca. }\end{array}$ & NO & $\begin{array}{c}\text { II/53. Wer- } \\
\text { theim } \mathrm{T} \text {. }\end{array}$ & $\begin{array}{l}7 \text { mes. } \\
\text { 3ien. Si,a } \\
\text { 3a. apzr. }\end{array}$ & Menopausia de un año. \\
\hline $\begin{array}{l}\text { No } 41 \\
30433 \\
\text { II } / 53\end{array}$ & $\begin{array}{c}27 \\
7 \\
17 \text { mes. }\end{array}$ & $\begin{array}{l}\text { E: II } \\
\text { Vag. Paramet. } \\
\text { Metrorragias. } \\
\text { T. Exofítico. }\end{array}$ & $\begin{array}{l}\mathrm{II} / 53 \\
\text { moso. } \\
\text { ción. }\end{array}$ & $\begin{array}{l}\overline{\text { Ca. esca- }} \\
\text { moso. } \\
\text { G: Posit. } \\
\text { Pólipo } \\
\text { degener. }\end{array}$ & $\begin{array}{l}\text { Fístula } \\
\text { vésico- } \\
\text { vaginal } \\
\text { pielocititis }\end{array}$ & $\begin{array}{l}\text { II } / 53: \text { Wer- } \\
\text { theim } \mathrm{T} \text {. }\end{array}$ & $\begin{array}{l}4 \text { mes. } \\
\text { mal. Con } \\
\text { reproduc. }\end{array}$ & $\begin{array}{l}\text { A los cuatro meses carcinomatosis, intra- } \\
\text { table. Probable muerte por esta causa } \\
\text { aunque no fue notificada al Instituto. Fís- } \\
\text { tula vésico-vaginal. }\end{array}$ \\
\hline $\begin{array}{c}\text { No } 42 \\
31549 \\
\text { VII } / 53\end{array}$ & $\begin{array}{r}46 \\
46 \\
9\end{array}$ & $\begin{array}{l}\text { E: II } \\
\text { Parametrios } \\
\text { Metrorragias. } \\
\text { T. Exofítico. }\end{array}$ & $\begin{array}{l}\text { Ca. Esca- } \\
\text { moso. } \\
\text { IX } / 53 \\
\text { Sin Ca. } \\
\text { III } / 54 \\
\text { Ca. Esca- } \\
\text { moso. }\end{array}$ & $\begin{array}{l}\text { Ca. esca- } \\
\text { moso. } \\
\text { G: no se } \\
\text { hicieron } \\
\text { cortes. }\end{array}$ & No & $\begin{array}{l}\text { VIII/53: Wer- } \\
\text { theim T. } \\
\text { IV } / 54 \text { Radium } \\
\text { vaginal. }\end{array}$ & $\begin{array}{l}8 \text { mes. } \\
\text { reprodic. } \\
\text { en cúpula } \\
12 \text { mes. } \\
\text { Bien. Sin } \\
\text { Ca. apar. }\end{array}$ & $\begin{array}{l}\text { Sospecha de reproducción en cúpula al } \\
\text { mes. Bs. negativa confirmada a los ocho } \\
\text { meses se trató con radium. Cuatro meses } \\
\text { después sigue bien. }\end{array}$ \\
\hline
\end{tabular}




\begin{tabular}{|c|c|c|c|c|c|c|c|c|}
\hline $\begin{array}{l}\text { CASO } \\
\text { HIST. No } \\
\text { FECHA }\end{array}$ & $\begin{array}{l}\text { EDAD } \\
\text { PARID. } \\
\text { LAPSO } \\
\text { A 10 } \\
\text { CONSUL. }\end{array}$ & $\begin{array}{l}\text { ESTADO SINTO- } \\
\text { MATOLOGIA }\end{array}$ & BIOPSIAS & $\begin{array}{l}\text { PIEZA Q. } \\
\text { GANG. }\end{array}$ & $\begin{array}{l}\text { COMPLIC. } \\
\text { OPERAT. }\end{array}$ & TRATAMIENTOS & CONTROL & OBSERVACIONES \\
\hline $\begin{array}{l}\text { No } 43 \\
32095 \\
\mathrm{IX} / 53\end{array}$ & $\begin{array}{c}53 \\
3 \\
3 \text { mes. }\end{array}$ & $\begin{array}{l}\text { E: II } \\
\text { Parametrios } \\
\text { Metrorragias. } \\
\text { T. Exofítico }\end{array}$ & $\begin{array}{l}\text { XI/52 } \\
\text { Ca. Esca- } \\
\text { moso. }\end{array}$ & $\begin{array}{l}\text { Ca. esca- } \\
\text { moso. } \\
\text { G: Negat. }\end{array}$ & NO & $\begin{array}{c}\mathrm{X} / 53 \text { : Wer- } \\
\text { theim } \mathrm{T} \text {. }\end{array}$ & $\begin{array}{l}12 \text { mos. } \\
\text { Bien. Sin } \\
\text { Ca. apar. }\end{array}$ & $\begin{array}{l}\text { Menopausia de siete años. Tratamiento } \\
\text { quirúrgico de fístula vésico-vaginal con } \\
\text { fracaso de éste. }\end{array}$ \\
\hline $\begin{array}{l}\text { No } 44 \\
33471 \\
\text { III } / 54\end{array}$ & $\begin{array}{c}57 \\
\quad 0 \\
4 \text { mes. }\end{array}$ & $\begin{array}{l}\text { E: II } \\
\text { Parametrios } \\
\text { y Ca. de cuerpo. } \\
\text { Metrorragias } \\
\text { T. Exofítico. }\end{array}$ & $\begin{array}{c}\text { III } / 54 \\
\text { adeno } \\
\text { Ca. de } \\
\text { cuello } \\
\text { y cuerpo. }\end{array}$ & $\begin{array}{l}\text { Sin Ca. } \\
\text { G: Negat. } \\
\text { Ca. Esca- } \\
\text { moso. }\end{array}$ & $\begin{array}{c}\text { Fistula } \\
\text { Vésico- } \\
\text { vaginal } \\
\text { esfacele } \\
\text { de cúpula } \\
\text { Pielocis. }\end{array}$ & $\begin{array}{l}\text { V/54: Radium } \\
\text { VI } / 54 \text { : Wer- } \\
\text { theim T. } \\
\text { VIII/54: repara- } \\
\text { ción de fístula. }\end{array}$ & $\begin{array}{l}4 \text { mes. } \\
\text { Bien. Sin } \\
\text { Ca. apar. } \\
\text { con fís- } \\
\text { tula. }\end{array}$ & \\
\hline $\begin{array}{l}\text { No } 45 \\
33535 \\
\text { IV } / 54\end{array}$ & $\begin{array}{c}38 \\
\quad 7 \\
3 \mathrm{mes} .\end{array}$ & $\begin{array}{c}\text { E: I } \\
\text { Parametrios } \\
\text { Metrorragias } \\
\text { F lujo con sang. } \\
\text { T. Exofítico. }\end{array}$ & $\begin{array}{l}\text { IV } / 54 \\
\mathrm{Ca} \text { esca- } \\
\text { moso. } \\
\mathrm{G}: \quad \text { Negat. }\end{array}$ & & $\begin{array}{l}\text { Hematoma } \\
\text { sub-perito- } \\
\text { neal. }\end{array}$ & $\begin{array}{c}\text { IV } / 54: \text { Wer- } \\
\text { theim T. } \\
\text { VI/54: exploración } \\
\text { y drenaje de quiste } \\
\text { subperitoneal }\end{array}$ & $\begin{array}{l}3 \text { mes. } \\
\text { Bien. Sin } \\
\text { Ca. apar. }\end{array}$ & $\begin{array}{l}\text { A los dos meses de tratamiento quirúr- } \\
\text { gico quiste multilocular (subperitoneal) } \\
\text { de contenido seroso sobre la zona de los } \\
\text { vasos iliacos izquierdos. Se drenó quirúr- } \\
\text { gicamente. Hidronefrosis bilateral mode- } \\
\text { rada a los dos meses de intervenida. } \\
\end{array}$ \\
\hline & & & & & & & & \\
\hline
\end{tabular}




\section{COMENTARIOS}

De los 45 casos presentados, uno se considera con diagnóstico equivocado y fue operado a causa de una biopsia remitida como positiva para carcinoma y tomada fuera del Instituto. Una biepsia tomada en el Instituto dio como resultado "Cervicitis Crónica" y una Citología fue considerada positiva para carcinoma. Posteriormente se confirmó el error de la primera biopsia.

Respecto a la edad de las enfermas, la de menor edad fue de ventisiete años y de setenta y seis la más vieja. La mayor incidencia de Carcinoma del cuello se encontró en la tercera década de vida (17 casos), siguiendo en su orden la cuarta década (13 casos) y la quinta (11 casos).

El lapso entre la probable iniciación de la sintomatología y la primera consulta en el Instituto es un dato muy relativo falseado por el factor de la apreciación subjetiva de la enferma y del médico examinador. Sin embargo anotado cuidadosamente en las historias orienta sobre la demora en consultar o en conseguir un diagnóstico apropiado.

El dato de paridad es interesante: 3 de las enfermas eran nuliparas, 1 había tenido un solo parto y 41 , es decir, ei $91 \%$, eran multíparas; de estas 29 habían tenido 5 o más partos.

En cuanto al aspecto del cuello a la inspección clínica, 27 casos, o sea el $60 \%$, fueron descritos como carcinomas exofíticos (coliflor, vegetante, exofítico); 12 , o sea el $26 \%$, como endofíticos (ulceración, erosión, cráter); 4, o sea un $8 \%$, como pólipos probablemente degenerados y 2 , o sea un $4 \%$, no fueron descritos claramente. Es interesante anotar que los 4 casos descritos como pólipos del cérvix, 3 fueron clasificados por el histopatólogo como "Carcinomas de Transición" y 1 como "Adenocarcinoma del cuello" (primitivo); se puede pensar con fundamento que la lesión primitiva fue un pólipo que degeneró posteriormente.

Histológicamente fueron clasificados 32 casos, es decir, el $73 \%$, como carcinomas escamosos (o epidermoides); 5, es decir, e: $11,1 \%$, como adenocarcinomas primitivos del cuello; 2, o sea el $4,4 \%$, como adenocarcinomas de cuello y cuerpo sin que pueda determinarse su localización primitiva aunque probablemente fue $\in \mathrm{n}$ el endometrio; 5 , o sea el $11,1 \%$, como carcinoma de transición. No se incluye el falso positivo. 
Conviene anotar los resultados del estudio de las piezas quirúrgicas, a pesar de que infortunadamente por distintas circunstancias no están anotados o no fueron practicados en su totalidad. Fueron clasificadas como sigue: 36 casos con carcinoma, de los cuales 1 , a pesar de tratamiento previo con radium, presentaba carcinoma residual en endometrio; 5 casos sin carcinoma (4 con tratamiento previo de radium y uno con amputación previa del cuello uterino); 3 sin estudio de pieza quirúrgica y el caso falso-positivo en el cual no se encontró tumor. Respecto al estudio de los ganglios en 41 Wertheims con linfadenectomía, 27 fueron negativos para carcinoma; 7 positivos y 7 sin estudio. De los 33 casos estudiados (se descarta el falso-positivo) los 7 positivos dan un porcentaje de $21,2 \%$ ligeramente más alto del anotado por otros autores; de estos 7 casos positivos, 5 se encontraron en Estados II; debe anotarse que en el caso número 16 se encontraron ganglios altos que no fue posible extraer por falta de sangre y mal estado de la enferma, por lo cual se aplicaron Rx. profundos posteriormente.

No se practicó linfadenectomía en 4 casos; en 2 de los cuales se hizo histerectomía vaginal tipo Schauta; en los otros 2 se había aplicado Radium previo. En uno de los casos tratados por vía vaginal, reproducción del carcinoma en ganglios y cúpula a los tres años y siete meses, que fue tratada con Rayos $\mathrm{X}$.

En 5 casos se aplicó radium previo (4 con adenocarcinoma del cuello y probablemente del cuerpo y uno con carcinoma escamoso de endocérvix). 4 de estos casos con pieza quirúrgica negativa para tumor.

En 6 casos se aplicaron irradiaciones posteriores al tratamiento quirúrgico (en 2 inmediatamente y en 4 posteriormente por reproducción o metástasis).

En cuanto a las complicaciones post-operatorias, se presentaron en 22 casos, o sea el 49\%. En 7 casos shock operatorio (4 irreversibles con muerte de las enfermas), debe anotarse que uno de estos casos causó la muerte tan sólo diez días más tarde por nefritis atribuible al mismo shock. En 7 casos, es decir 15,5\%. se presentaron fístulas urinarias ( 1 uretero-vaginal y 6 vésicovaginales), correspondieron 5 a Estados II y 2 a Estado I, con un porcentaje en Estado II de $21.7 \%$. La fístula uretero-vaginal cưó por intervención quirúrgica, una de las fístulas vésico-vaginales curó con cateterismo ureteral e injertos de placenta; otras 2 tratadas quirúrgicamente se reprodujeron. 
Si se consideran separadamente los Estados I y II, se encuentra en Estado I 36,3\% casos con complicaciones y en Estado II $60,8 \%$.

La mortalidad fue semejante para los dos estados clínicos y esto era de esperar, puesto que el traumatismo quirúrgico es semejante y el estado general de las enfermas no suele diferir.

Las otras complicaciones no numeradas hasta ahora fueron 2. casos de hematoma pélvico, parálisis vesical prolongada en 2 casos y parálisis de brazo derecho de origen mecánico 2 casos. No detallan otras complicaciones de menor importancia que se encuentran en el cuadro general.

En conjunto se pueden dar los siguientes datos sobre el control de los casos estudiados:

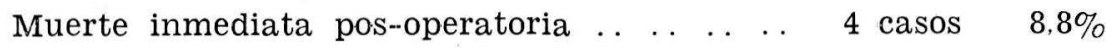
Muerte por carcinomatosis confirmada .. .. 4 casos $8,8 \%$ Perdidas de control desde intervención .. .. 6 casos $13,3 \%$ Perdidas de control posteriormente ....... 12 casos $26,6 \%$

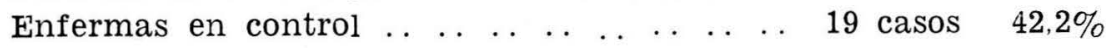
Total pérdidas de control . . . . . . . . . . . . . 18 casos $40 \%$ Reproducciones o metastásis antes 1 año... 10 casos 22,2\%

De las enfermas actualmente en control, una presenta carcinomatosis y lleva 12 meses de operada, la otra con reproducción en cúpula a los 8 meses que se trató con radium lleva 4 meses de control sin carcinoma aparente; las 17 restantes sin carcinoma aparente, de las cuales 6 con tres años o más de supervivencia ( 3 con más de cuatro años) sin carcinoma aparente. Una de las enfermas perdidas de control duró tres años sin carcinoma aparente y presentó a los tres años y siete meses reproducción; ya se habló de este caso tratado por la técnica de Schauta. De los 10 casos ('ontrolados con reproducción, 8 se presentaron en enfermas ciasificadas en Estado II.

\section{CONCLUSIONES}

I) - En los casos estudiados se puede afirmar que la morbilidad, y frecuencia de las reproducciones post-operatorias es mucho mayor en los casos clasificados clínicamente como Estados II. Es especialmente significativa la mayor incidencia de fístulas urinarias.

II) -El número de casos con ganglios metastásicos es también significativamente más alto en los Estados II. 
III) - La muy alta morbilidad anotada por los Estados II $(60,8 \%)$ justifica el cambio de conducta acordado en el Servicio Ginecológico del Instituto Nacional de Cancerología o sea remitir a tratamiento con Radium y Roengenterapia profunda los casos en este estado clínico, siempre y cuando el tratamiento por irracliación pueda aplicarse en su totalidad.

IV) - Cuatro de los casos presentados puede decirse con fundamento que se originaron en pólipos del cérvix presentando un cuadro histopatológico de "Carcinoma de Transición". Esto permite insistir sobre la conveniencia de tratamiento apropiado y examen anatomopatológico de los pólipos de cuello uterino.

V) - La alta incidencia de carcinoma del cuello en enfermas niultíparas (91\% de los casos en enfermas multíparas) está en favor de factores predisponentes o desencadenantes de carcinoma por los repetidos traumatismos del parto; es un argumento en favor de la conveniencia de controles ginecológicos periódicos en este tipo de enfermas.

VI) -El porcentaje de enfermas perdidas para control es muy alto $(40 \%)$ y puede atribuirse por una parte a ignorancia de éstas y de sus familiares y por otra a factores económicos, que no permiten el regreso a control por el gasto que supone el viaje y permanencia en la ciudad cuando viven fuera de ella. El Instituto cita periódicamente a sus enfermos mediante cartas dirigidas a éstos y a sus familiares.

VII) - Se considera que cualquier otro tratamiento quirúrgico menos radical que el Wertheim Taussig es inútil y generalmente perjudicial para las enfermas, y que cuando se encuentran adherencias firmes de vejiga a útero, de recto a útero o vagina, o ganglios para-aórticos sospechosos de metastasis, el cirujano debe tener el valor de cerrar la herida abdominal y remitir la enferma a tratamiento radioterápico.

VIII) - Se puede pensar que algunas de ias fístulas vésico-vaginales se han sucedido por falta de irrigación y que es conveniente no ligar la arteria vesical terminal de la hipogástrica, sino ligar la uterina a la salida de la hipogástrica, con el fin de conservar una mejor irrigación vesical. Esta modificación ya ha sido adoptada para algunos casos en el Instituto. 


\section{BIBLIOGRAFIA}

ALJURE M. FOSTUNATO.- El Tratamiento Quirúrgico del Carcinoma del Cérvix Uterino. Tesis de Grado. Universidad Nacional. Bogotá, Colombia, noviembre, 1953.

LASH A. F.- Carcinoma of Cervix Uteri. S. Clin. North America 33: 231-240, febrero, 1953.

GRAHAM JOHN and MEIGS JOSE V.-Residual Carcinoma in Vaginai Cuff after Radical Hysterectomy with Bilateral Pelvic Lymph Node Dissection. Am. J. Obst. \& Gynec. 64: 402-403, Aug, 1952.

NOVAK EMIL: Carcinoma of the Cervix. Gynecologic and Obstetric Pathology. Third edition. Ed. Saunders Philadelphia and London. P. 109. 1952. 